\section{EMBRYAIDDLE Aeronautical University}

SCHOLARLY COMMONS
International Journal of Aviation, Aeronautics, and Aerospace

\title{
Detecting and Assessing Collision Potential of Aircraft and Small Unmanned Aircraft Systems (sUAS) by Visual Observers
}

\author{
Samuel M. Vance \\ Oklahoma State University - Main Campus, matt.vance@okstate.edu \\ Ryan J. Wallace \\ Polk State College, ryan.wallace@erau.edu \\ Jon M. Loffi \\ Oklahoma State University - Main Campus, jon.loffi@okstate.edu \\ Jamey D. Jacob \\ Oklahoma State University, jdjacob@okstate.edu \\ Jared C. Dunlap \\ Oklahoma State University - Main Campus, jared.dunlap@okstate.edu \\ Taylor A. Mitchell \\ Oklahoma State University, taylor.mitchell@okstate.edu
}

Follow this and additional works at: https://commons.erau.edu/ijaaa

Part of the Aviation Commons

\section{Scholarly Commons Citation}

Vance, S. M., Wallace, R. J., Loffi, J. M., Jacob, J. D., Dunlap, J. C., \& Mitchell, T. A. (2017). Detecting and Assessing Collision Potential of Aircraft and Small Unmanned Aircraft Systems (sUAS) by Visual Observers. International Journal of Aviation, Aeronautics, and Aerospace, 4(4). https://doi.org/10.15394/ ijaaa.2017.1188

This Article is brought to you for free and open access by the Journals at Scholarly Commons. It has been accepted for inclusion in International Journal of Aviation, Aeronautics, and Aerospace by an authorized administrator of Scholarly Commons. For more information, please contact commons@erau.edu. 
The purpose of a visual observer is to assist a sUAS operator or remote pilot in exercising see and avoid responsibilities to avoid collision hazards. According to $14 \mathrm{CFR} \S 107.31$, remote pilots and visual observers share responsibility for knowing the location, altitude, attitude, and flight direction of the sUAS, observing the airspace for traffic and other hazards and, preventing the creation of a hazard to people or property. In performing these responsibilities, visual observers must coordinate with the remote pilot or sUAS operator to "scan the airspace where the sUAS is operating for any potential collision hazard and maintain awareness of the position of the small unmanned aircraft through direct visual observation" (14 CFR $\S 107.33)$.

\section{Problem}

While visual observers are not required to be employed by sUAS operations, their use is encouraged as a regulatory method of complying with 14 CFR $\S 107.31$ provisions. Currently, there is little existing evidence of the effectiveness and accuracy of visual observers in detecting and evaluating the collision potential of invading air traffic.

\section{Purpose}

The purpose of this study was to perform experimental, human factors research to determine the effectiveness of sUAS visual observers in detecting a general aviation aircraft collision hazard with an unmanned aircraft. The study sought to evaluate visual observer effectiveness at detecting a closing general aviation aircraft. Additionally, researchers sought to assess the accuracy of visual observer altitude, range, and closure rate estimates to inbound aircraft. This data was used to establish operational recommendations to improve visual observer performance.

\section{Research Questions}

This study sought to answer the following research questions:

1. At what range do visual observers reliably detect invasive aircraft?

2. How accurate are visual observers at estimating distance to aircraft?

3. How accurate are visual observers at estimating closure rate of an aircraft?

4. Does the error between visual observer estimates and actual aircraft closure rate provide adequate time for a remote pilot to initiate evasive maneuvers before a potential collision? 
5. What human factors considerations affect visual observer detection and assessment of aircraft collision potential

\section{Literature Review}

\section{Human Visual Factors Affecting Visual Observers}

Williams and Gildea (2014) highlight four human factors issues that affect visual observer performance.

Foveal Vision. Foveal vision addresses an individual's ability to discern details that are used to derive symbolic information from images (Williams \& Gildea, 2014). The fovea encompasses only about one degree of the human field of view. Normal human vision acuity within the foveal view is estimated to be approximately 0.167 of a degree, giving an object with a small $1 \mathrm{ft}^{2}$ cross sectionsuch as a small UAS--a theoretical maximum visual range of 3,438 feet (Williams $\&$ Gildea, 2014). Foveal vision is adversely affected by certain environmental conditions, such as low light levels or poor contrast (Williams \& Gildea, 2014). Degraded foveal vision can also be caused by various physical conditions including a lack of sleep, hypoglycemia, or hypoxia (Williams \& Gildea, 2014). Substances including alcohol or tobacco can also diminish visual acuity in the fovea (Williams \& Gildea, 2014).

Visual Accommodation. This process describes how the eyes refocus between objects at varying ranges (Williams \& Gildea, 2014). The responsiveness of visual accommodation can be affected by age or fatigue (Williams \& Gildea, 2014). Additionally, visual accommodation can be hindered by a lack of objects within the field of view, which causes the eyes to naturally reset their focus at a default position in front of the viewer, known as empty-field myopia (Williams \& Gildea, 2014).

Peripheral Vision. Peripheral vision refers to all vision that falls outside the foveal view, and generally spans 120 degrees of vertical and 190 degrees of lateral vision (Diffrien, Tilley \& Harman, 1981, as cited in Williams \& Gildea, 2014).

Vigilance. Although not directly related to human visual physiology, the impact of human vigilance has a substantial impact on the effectiveness of visual detection and recognition. Boff \& Lincoln (1988) determined that visual scanning performance degrades over the course of 30-60 minutes, as a result of boredom or fatigue (as cited in Williams \& Gildea, 2014). Several other factors are likely to 
affect attention and vigilance. Parasuramn \& Davies (1977) codify a few of these factors to include: the rate of the occurrence of the stimulus or event rate, task difficulty, signal duration or intensity, and several other influencing variables (as cited in Williams \& Gildea, 2014).

Williams and Gildea (2014) conclude that in spite of the capabilities of human vision, "[it] is often unreliable, even under the most ideal conditions" (p. 2).

\section{Related Studies}

Crognale (2009) conducted a series of experiments to test the ability of ground-based visual observers to visually detect an approaching unmanned aircraft. A Scan Eagle UAS approached the visual observers at scripted cardinal directions at a distance of approximately one mile. To prevent observers from acquiring the UAS using audio cues, participants wore ear plugs. During the test, participants successfully detected the UAS platform during 97\% $(n=224)$ of the 240 trials at a mean distance of 327 meters. Crognale further determined that visual observer detection was required to be made at a minimum of 12 seconds--a distance of 300 meters based on the Scan Eagle's speed--to allow a remote operator adequate time to recognize the potentially unsafe condition and perform an evasive maneuver to avoid collision. Based on data from Crognale's experiment, participants only detected the Scan Eagle UAS greater than 12 seconds from intercept on $49 \%(n=$ 118 ) out of the 240 trials.

The second of Crognale's (2009) experiments evaluated each participant's ability to accurately estimate UAS distance and altitude. The UAS maneuvered in a scripted pattern around the flying site, orbiting at defined points. At each orbiting point, the visual observers would attempt to judge the distance and altitude of the unmanned aircraft. Orbiting points were placed at quarter-mile intervals in distance ranging from $0.25 \mathrm{SM}$ to $0.75 \mathrm{SM}$, and 500-foot increments in altitude from 500 feet to 1,500 feet AGL. On average, participants tended to overestimate distance by $40 \%$ and altitudes by $60 \%$.

In Crognale's (2009) third experiment, visual observers were asked to follow the UAS until it was no longer visible. The Scan Eagle was then vectored along a reverse course until the visual observer reacquired the UAS. Participants lost visual contact with the UAS at a mean distance of 1,276 meters and reacquired the aircraft at a mean distance range of 898 meters. 


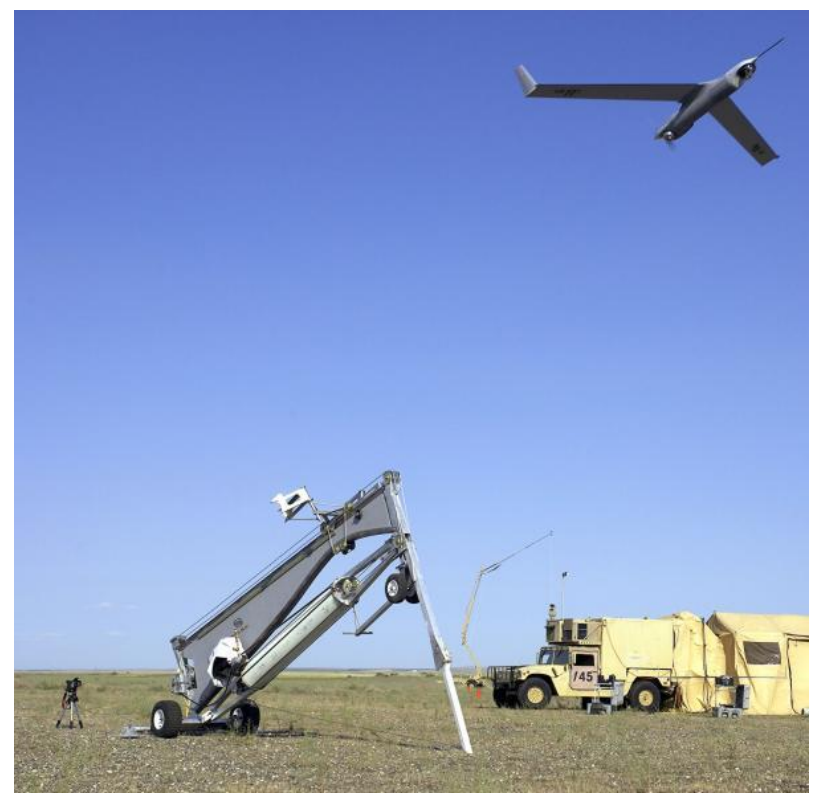

Figure 1. Scan Eagle UAS. Public domain image obtained from http://www.af.mil/About-Us/FactSheets/Display/Article/104532/scan-eagle/. The Scan Eagle UAS typically operates under 15,000 feet, and sports a 5-foot long fuselage with a wingspan of 10 feet. Reference platform details at: http://www.boeing.com/history/products/scaneagle-unmanned-aerial-vehicle.page

In Crognale's (2009) fourth experiment, visual observers were asked to assess the collision potential between the Scan Eagle and an intruding aircraft. Crognale described challenges in executing the experimental protocol of this final exercise. One notable finding was that visual observers were unable to assess collision potential unless they were able to see both the aircraft and UAS at the same time.

Crognale (2009) concluded that visual observers performed relatively poorly at visually acquiring UAS platforms if their inbound position was uncertain. Conversely, visual observers performed better when the inbound position of the aircraft was known or confined to a smaller region or arc. Crognale suggests that relative motion was also likely to improve aircraft target detection. Perhaps Crognale's most notable finding was that "visual detection by observers in uncertain situations (without sound cues, TCAS, or radio announcements) is unlikely to contribute significantly to collision avoidance" (Crognale, 2009, p. 67).

Dolgov et al. (2012) conducted a field study of UAS visual observers to assess their performance during varying periods of the day and night (as cited in Williams \& Gildea, 2014). Similar to the Crognale (2009) study, Dolgov et al. (2012) assessed visual observers' abilities to accurately assess collision potential between manned and unmanned aircraft. Dolgov determined that visual observers 
performed better in nighttime conditions, with participants detecting aircraft at further distances over daytime. Dolgov assessed visual observer assessment of collision potential as poor, with wide variability among study participants.

A subsequent study by Dolgov (2016) was performed to examine "visual observers' ability to maintain line of sight with a light-sport manned aircraft and a small unmanned aircraft system (sUAS: Raven RQ-11B or Wasp III) and predict imminent collisions between them" (Dolgov, 2016, p. 1). Three participants conducted a series of detection and tracking experiments for three sUAS craft. The research assessed visual observer performance under both night and dusk conditions. Results showed that visual observers were able to detect and track much better during night and dusk than during the day. Visual observers were able to maintain visual tracking for the Raven during $36.1 \%$ of the flight time during the daytime compared to the $29.5 \%$ for the Wasp. Visibility improved considerably during dusk to $83.9 \%$ for the Raven and $52.1 \%$ for the Wasp. Nighttime visibility was also improved with $73.6 \%$ overall visibility for the Raven and $75.0 \%$ for the Wasp. Detection distance of both the sUAS craft and light sport aircraft was also found to be generally greater at night. Dolgov (2016) noted that "Generally, the distance at which an aircraft can be seen in the daylight depends on its physical size and contrast against the sky and clouds, whereas, the distance at night depends on its lighting system" (Dolgov, 2016, p. 51).

\section{Method}

This research project was conducted as a field study, based in large part on the previous work of Crognale (2009) and Dolgov et al. (2012). Ten volunteer participants were recruited from a Part 141 collegiate flight training program in the Midwestern United States using a purposeful sampling technique.

Participants were instructed to serve as visual observers for an unmanned aircraft operation at a small $\mathrm{RC}$ flying airfield under the scenario of aiding a remote pilot in detecting and avoiding converging, conflict aircraft. The small unmanned aircraft was launched, controlled, and recovered by a qualified remote pilot operating in proximity to the participants. Participants were instructed to only interact with the assigned researcher in lieu of the actual remote pilot and were separated from control equipment and information displays to protect study integrity. Table 1 indicates the self-reported qualifications and visual acuity of the respective participants; unfortunately, individual audio acuity information was unavailable. 
UAS flights were conducted in accordance with the collegiate institution's Federal Aviation Administration Modernization and Reform Act of 2012 Section 333 Exemption and accompanying Certificate of Authorization. Researchers used a DJI Matrice 100 small unmanned aircraft equipped with supplemental STROBON 50mAh navigation strobe lights affixed to the sUAS platform's anterior surface. A manned Cessna $172 \mathrm{~S}$ aircraft was subsequently launched to stimulate participant responses. The manned aircraft performed a series of pre-scripted maneuvers to intercept the small unmanned aircraft in various relative configurations. The scripted maneuvers included a 200 -foot vertical differential between the aircraft and sUAS to simulate the perception of a near collision, without putting either platform in actual danger. The aircraft was equipped with G1000 avionics suite, which allowed researchers to extract timestamped geolocation data. Similarly, the sUAS craft was also indigenously capable of GPS tracking to collect similar location data.

Table 1.

Participant Aeronautical Demographics

\begin{tabular}{llll}
\hline Participant & FAA Pilot Certificate(s) & $\begin{array}{l}\text { Medical } \\
\text { Certificate }\end{array}$ & Reported Vision \\
\hline 1 & CPL & $3^{\text {rd }}$ Class & Unknown \\
2 & PPL & $3^{\text {rd }}$ Class & Unknown \\
3 & CFII & $3^{\text {rd }}$ Class & Unknown \\
4 & SP & $2^{\text {nd }}$ Class & $20 / 25$ \\
5 & CFI & $1^{\text {st }}$ Class & Unknown \\
6 & SP & $1^{\text {st }}$ Class & $20 / 20$ \\
7 & CFI & $1^{\text {st }}$ Class & Unknown \\
8 & PPL-IR & $1^{\text {st }}$ Class & $20 / 20$ \\
9 & PPL-IR & $1^{\text {st }}$ Class & $20 / 20$ \\
10 & PPL-IR & $3^{\text {rd }}$ Class & $20 / 20$ \\
\hline
\end{tabular}

Note: $(\mathrm{SP}=$ Student Pilot; PPL $=$ Private Pilot License; $\mathrm{IR}=$ Instrument Rating; $\mathrm{CPL}=$ Commercial Pilot license; $\mathrm{CFI}=$ Certified Flight Instructor; CFII $=$ Certified Flight Instructor-Instrument 

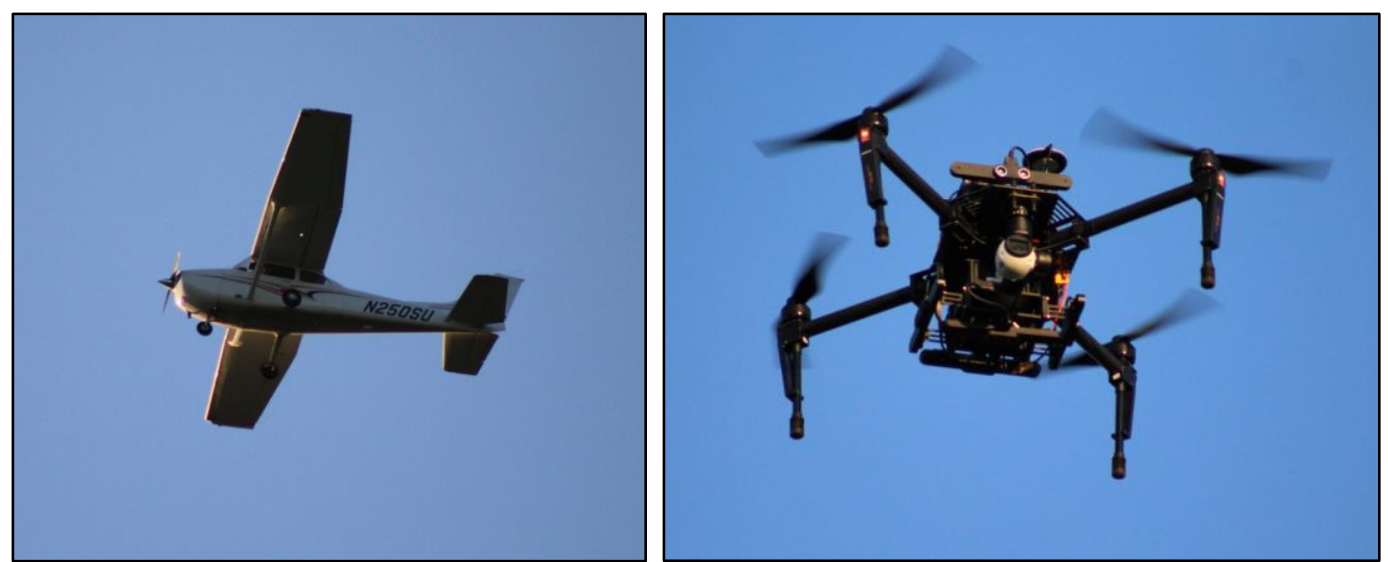

Figure 2. [LEFT] G-1000 equipped, Cessna 172/S aircraft (36-ft wingspan) used during the experiment. [RIGHT] DJI Matrice 100 (27-in width, rotor hub-to-rotor hub) outfitted with 50mAh STROBON Navigation Strobe Lights affixed to UAS anterior surface. STROBON technical information available from: http://www.flytron.com/led-systems/150-strobon-navigation-strobeblue.html

The following scripted intercepts were used during the experiment:

- Control Intercept (randomized): Manned aircraft flew inbound to sUAS flight location, however sUAS was not launched.

- Intercept 1: sUAS oriented slightly left of manned aircraft course

- Intercept 2: sUAS oriented slightly right of manned aircraft course

- Intercept 3: sUAS conducted a repeating lateral maneuver directly in front of manned aircraft

- Intercept 4: sUAS flew a head-on convergence course with the manned aircraft

Participants were asked to identify when they initially detected an inbound aircraft, either by hearing or sight. Once the aircraft was visually located, the participant was instructed to estimate its distance, altitude, and closure rate in seconds to intercept the sUAS. Finally, participants were asked to judge the lateral distance between the aircraft and sUAS at their closest point of approach. The exact time of each student response was recorded via a stopwatch synchronized to both the sUAS and G-1000 systems. Additionally, participants were asked to provide their perceptions on their ability to perform the requested detection and estimation tasks. Both quantitative and qualitative results were recorded by one of the members of the research team. 


\section{Assumptions \& Limitations}

The experiment was executed as described with the following assumptions and limitations.

The DJI Matrice 100 sUAS platform used during the experiment was operated in accordance with the institution's approved Section 333 exemption and Certificate of Authorization, which limited the sUAS altitude to a maximum of 400 feet AGL. While participants were asked to estimate altitude during each intercept, the altitudes of both the sUAS and aircraft were fixed at 400 feet AGL and 600 feet AGL, respectively. Researchers elected not to vary the altitude of the aircraft, since it would compromise a parallel visibility experiment that was being conducted aboard at the same time.

Accuracy of the collected geolocation data was based on the fidelity of both the Matrice 100's (non-SDK) indigenous GPS system as well as the aircraft's G1000 system. Coupled with data truncation methods used to simplify calculations, overall distance reporting interval accuracy was limited to approximately 53 feet.

Each participant was exposed to a single, randomized control test intercept in which the sUAS was not flown. This pass was not officially a part of the ground experiment, but was used to collect data for the parallel airborne experiment. The researchers assumed that exposure to this single intercept did not adversely taint or affect the ability of the visual observers to detect or estimate aircraft conditions during subsequent intercept passes. Visual observer range and altitude estimation data was still opportunistically collected for this intercept, however, was excluded for calculations relating to closure time and distance estimates.

Researchers anticipated sUAS faults and other unforeseen operational impacts to potentially affect the experiment. Researchers planned to repeat passes if malfunctions did not present a safety of flight issue for either the aircraft or sUAS platform.

\section{Results}

The experiment was conducted between July 10-14, 2017, between 18:0020:00 local time. Of the 40 planned intercepts, the experiment yielded 39 usable datasets for audio detection and 38 usable datasets for visual detection from more than 89,511 time-correlated GPS data points. Data was opportunistically collected during the control intercept passes, resulting in 10 additional datasets for initial detection range only. 


\section{Unanticipated Conditions}

Mechanical/operational conditions. The sUAS encountered a battery failure during Participant 6's run, requiring the fourth experimental intercept to be re-accomplished. Similarly, the airborne aircraft was forced to abort as a result of high winds, rain, and nearby convective activity during Participant 10's fourth intercept. Researchers were unable to repeat this intercept prior to concluding the experiment.

Experimental participant influences. Some participant actions resulted in invalidating data for various reasons. Participant 5 was able to maintain uninterrupted visual contact with the outbound aircraft following the conclusion of intercept 3, resulting in the invalidation of Participant 5's intercept 4 visual detection range data. Also, Participant 4 lost sight of the sUAS during intercept 2 making the participant unable to provide an aircraft-sUAS closure or distance estimate.

\section{Detection}

Participants detected the aircraft on $100 \%(n=49)$ of the intercepts, including the control intercepts. As depicted by Figure 3, audio detection of the aircraft ranged from 3,010 feet to 29,304 feet, with an average detection distance of 8,605.4 feet. For calculation purposes, outliers were retained, since it was not completely clear to researchers what was causing the variability in some participant's detection thresholds.

Visual detection of the aircraft ranged from a minimum of 1,637 feet to a maximum of 20,434 feet, with an average detection distance of 8,618.6 feet as depicted in Figure 4.

This performance was a substantial improvement over the Crognale (2009) results, where mean visual observer detection distance was determined to be only 327 meters [1,073 feet]. There is a strong likelihood that this variation can be explained by the fact that this study used a C-172, which has a substantially larger visible cross-section than the Scan Eagle UAS used in the Crognale (2009) study. 


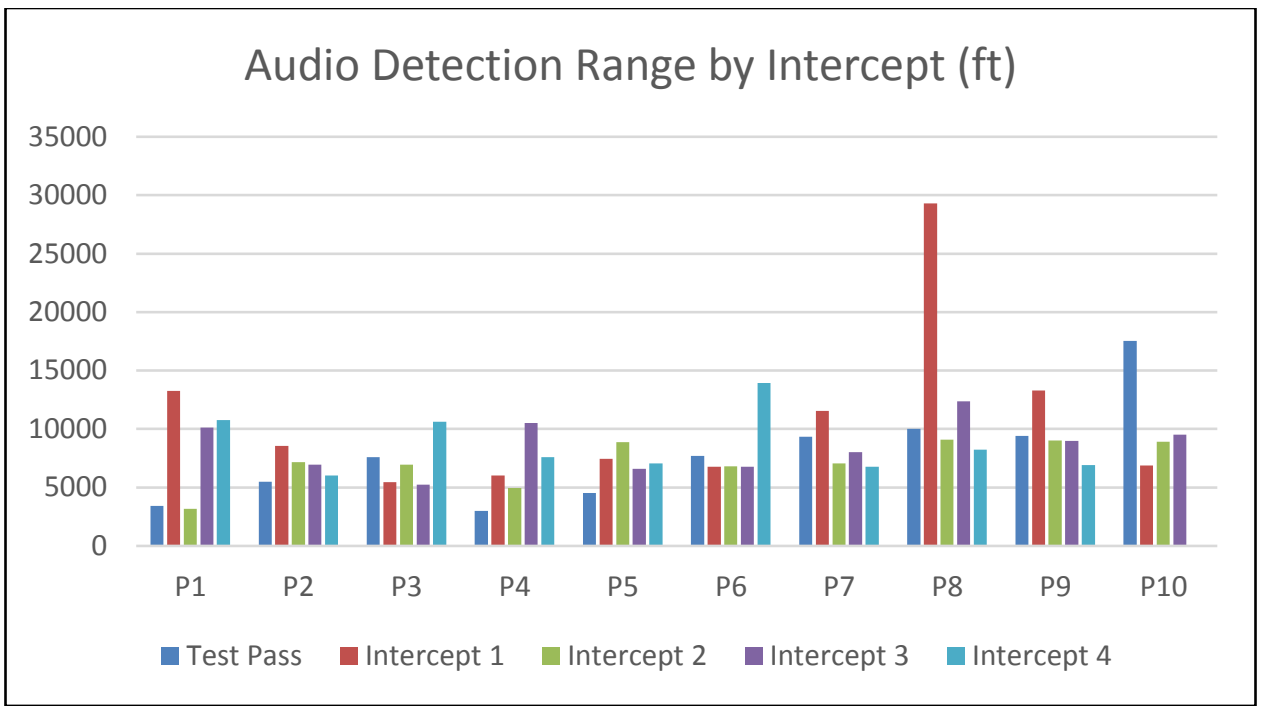

Figure 3. Audio detection range by participant and intercept, displayed in feet.

Participants initially detected the aircraft by auditory means during $30.5 \%$ of the intercepts $(n=18)$; and, initially by visual means during $27.1 \%$ of the intercepts $(n=16)$. Participants simultaneously detected the aircraft by both visual and auditory means during $32 \%$ of the intercepts $(n=19)$. Audio detection range generally preceded visual detection range by a mean of 159 feet. Results are displayed in Figure 5.

Since auditory detection was not a studied variable in either the Crognale (2009) or Dolgov et al. (2012) studies, it was not possible to provide comparison of these results to established research benchmarks. For most participants, however, it appeared that detection by one sensory modality was followed in short order by the second modality. It is notable that in some uncommon cases, delayed detection by the second sensory modality exceeded a half statute mile or more. This is particularly troubling in scenarios when the visual observer hears an aircraft but cannot rapidly visually acquire it to make a safety of flight or collision potential judgement.

\section{Estimation}

Once participants visually detected the aircraft, they were asked to estimate the duration until the sUAS and aircraft intercepted. Researchers documented 37 duration estimates from the available 40 experimental intercepts. Duration estimates ranged from 7 seconds to 120 seconds. Estimates were compared against the actual intercept time, which was calculated by determining the closest point of approach between the aircraft and sUAS GPS data, and correlated post-hoc to an 
intercept time. Actual intercept times varied from 13 seconds to 108 seconds, with a mean of 54 seconds. During 26 of the intercepts, participants overestimated the available duration to the aircraft-sUAS intercept; and, underestimated the duration during 11 of the aircraft-sUAS intercepts. The mean estimation error was determined to be 17 seconds. The results are displayed in Figure 6.

This was a somewhat disturbing finding, as the visual observer tendency towards overestimating available response time may result in delayed evasive maneuvers or collision avoidance. It should also be noted, that such evasive actions are not likely to occur instantaneously, as the visual observer not only needs to detect an intruding aircraft, assess the situation, and perceive a potential collision hazard, but also needs to communicate that information to the remote pilot. Given the relatively short intercept time from when initial visual detection is made, any further delay incurred from overestimation may not leave the remote pilot adequate time to react to evade a collision threat.

During each intercept, participants were asked to estimate the distance between the aircraft and the sUAS platform when they perceived that the two were closest to each other. Researchers documented 38 distance estimates ranging from 30 feet to 2,000 feet. These estimates were compared against the actual calculated distances based on a comparison of the aircraft and sUAS GPS location data. On 11 of the intercepts, participants underestimated the range between the aircraft and sUAS platform; whereas, on 27 of the intercepts, participants overestimated the range. The mean estimation error at the point of intercept was calculated to be 290 feet. Results are depicted in Figure 7. 


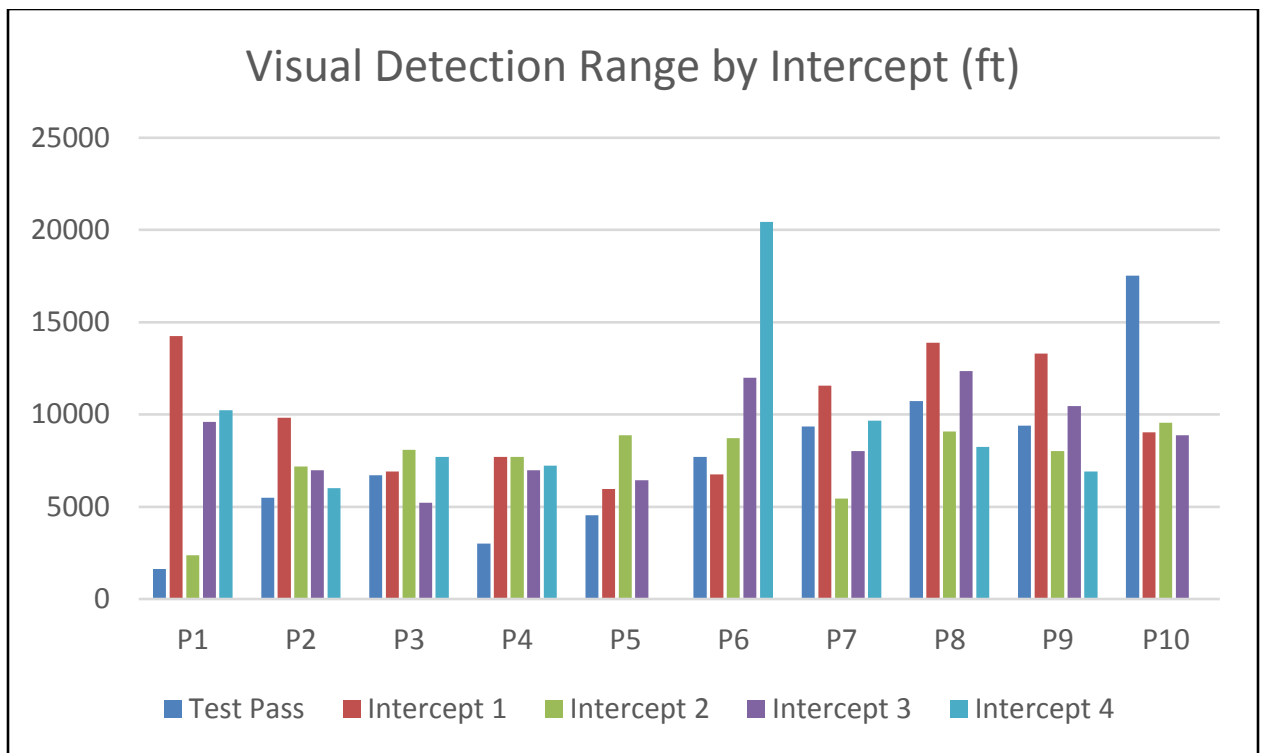

Figure 4. Visual detection range by participant intercept, displayed in feet.

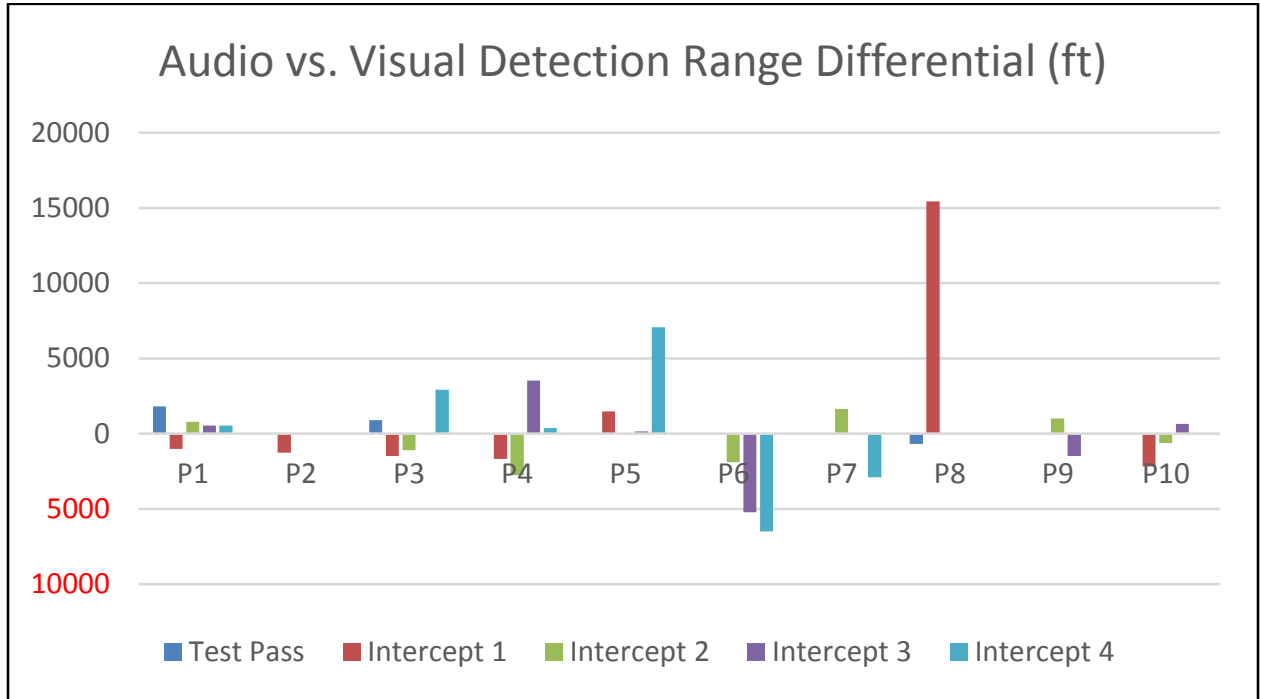

Figure 5. Audio vs. visual detection range differential by participant and intercept, displayed in feet. Graph depicts initial aircraft detection range with initial audio detection being displayed as positive values, and initial visual detection displayed as negative values. The elapsed range difference between when the participant detected the aircraft via one sensory modality until it was acquired via the other sensory modality is represented by the magnitude graphed on the Y-axis. 


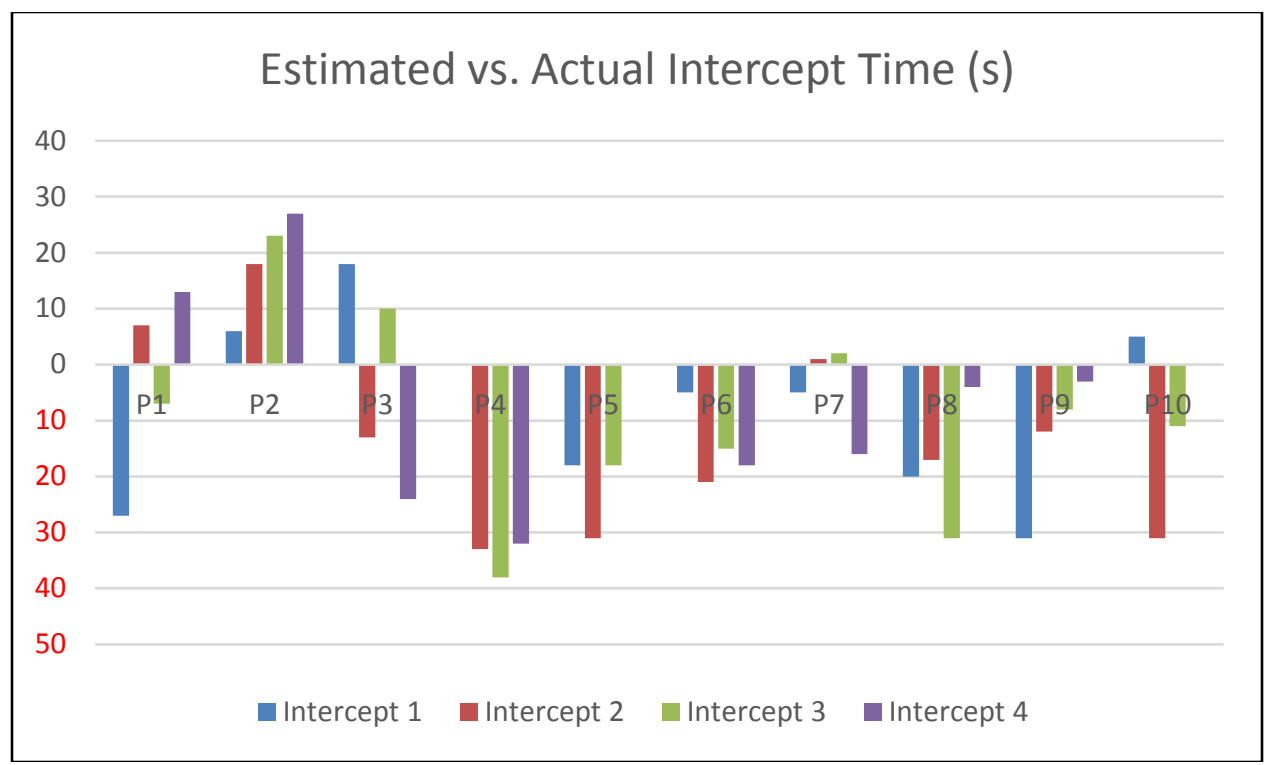

Figure 6. Estimated vs. actual intercept time by intercept, depicted in seconds. Graph depicts the differential between participant estimates of available duration between initial visual sighting and closest distance convergence between aircraft and sUAS platforms, or actual intercept time. Positive values represent an underestimate; whereas, negative values represent an overestimate.

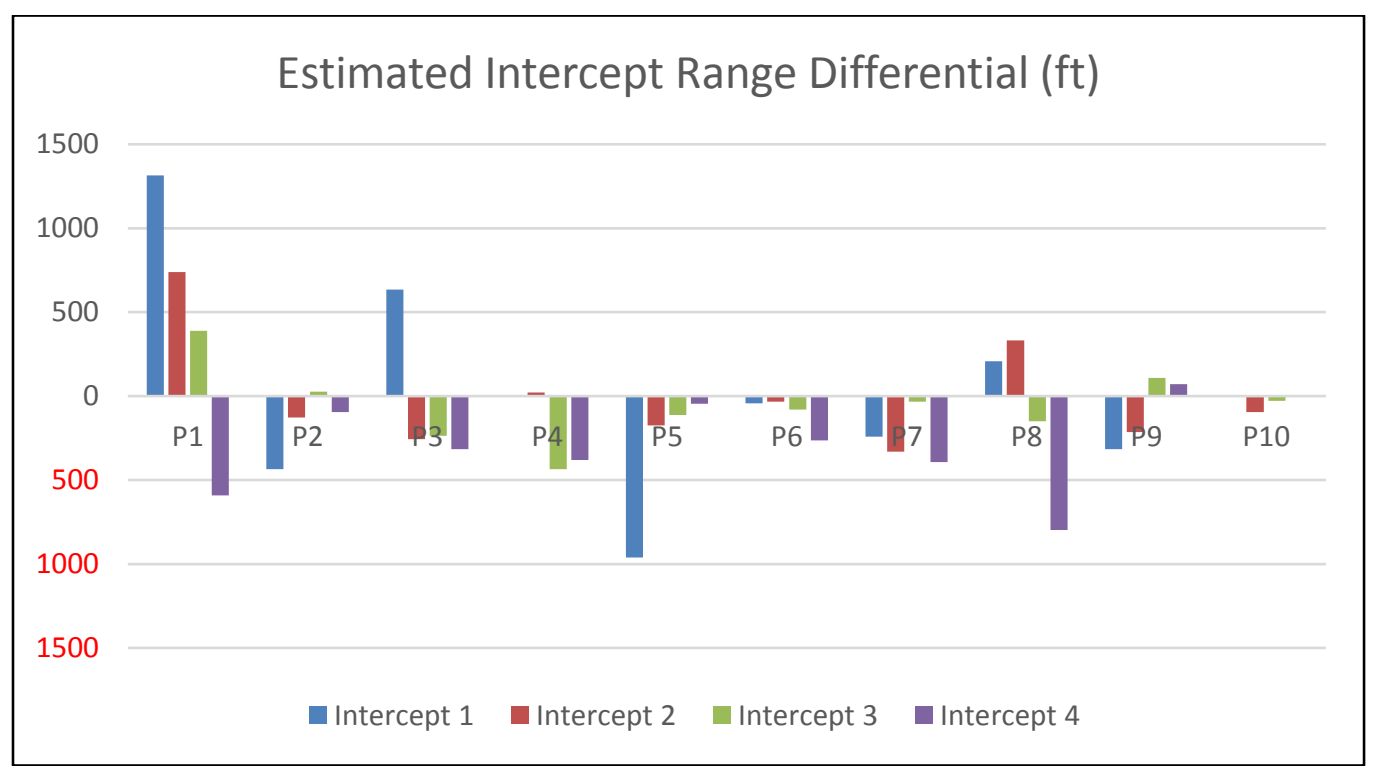

Figure 7. Difference between estimated and actual distance between aircraft and sUAS platform at closest intercept point, displayed in feet. Positive values indicate the participant underestimated the actual range. Negative values indicate the participant overestimated the range. The variability between participant estimates and actual distances are represented by the magnitude plotted on the Y-axis. 
Crognale's (2009) research seems to support the general preference of participants to overestimate distances. In Crognale's (2009) experiment "subjects seemed to slightly overestimate distances, particularly at longer distances," however, when error is calculated as a percentage of the distance, the error rate decreases slightly with increased distance (p. 40). Crognale (2009) calculated the error rate, dubbed root mean square error (RMSE) as ranging from $20 \%$ to $90 \%$, with a mean of $39.4 \%$. While the authors of this study did not tabulate estimation error in the same terms, the wide variability and poor estimation accuracy is apparent and consistent with the Crognale (2009) study.

\section{Qualitative Findings}

Upon conclusion of each intercept, participants were asked about their perceptions regarding their ability to detect aircraft and provide distance estimates to facilitate sUAS collision avoidance. Participant comments and researcher field notes were compiled and qualitatively analyzed for commonality, using a concept analysis technique. The following recurrent themes were identified:

Platform size differential affected distance estimates. Four of the 10 participants indicated that the size differential between the aircraft and sUAS platform adversely affected their ability to accurately judge distances. Participant 2 stated, "it was hard to accurately estimate distances due to size differences." Participant 3 echoed these comments stating that estimates were "not very accurate due to size disparity." Participant 8 indicated that the size disparity also affected their ability to determine vertical differential stating, "it is harder then it seems...the aircraft appears lower due to the large size, while the sUAS appears higher due to the small size - perhaps, similar to a sloped runway illusion encountered by pilots."

This finding is not altogether unsurprising. The overall lack of skyward objects to provide relative size and distance comparisons is a fairly well-known phenomenon. Figure 8 illustrates this optical illusion.

Discerning altitude differences and collision potential was difficult. Although operational constraints prevented researchers from further exploring this aspect of visual detection and judgement, several participants commented. Particpant 1 said, "Both vehicles look really close at the same altitude...it was very difficult to tell differing altitudes. Participant 4 also described the difficulties associated with altitude discernment, as being "very had to estimate distance and altitude between the vehicles." Participant 5 similarly explained, "it was very hard to estimate collision...estimating laterial separation was easier than estimating height differences." Participant 6 echoed similar experiences stating, "It is hard to 
tell perspectives and exact angles or altitudes from the relative motion of the objects." Participant 10 also found altiutude estimation difficult stating, "Vertical distance was the most difficult to estimate..."

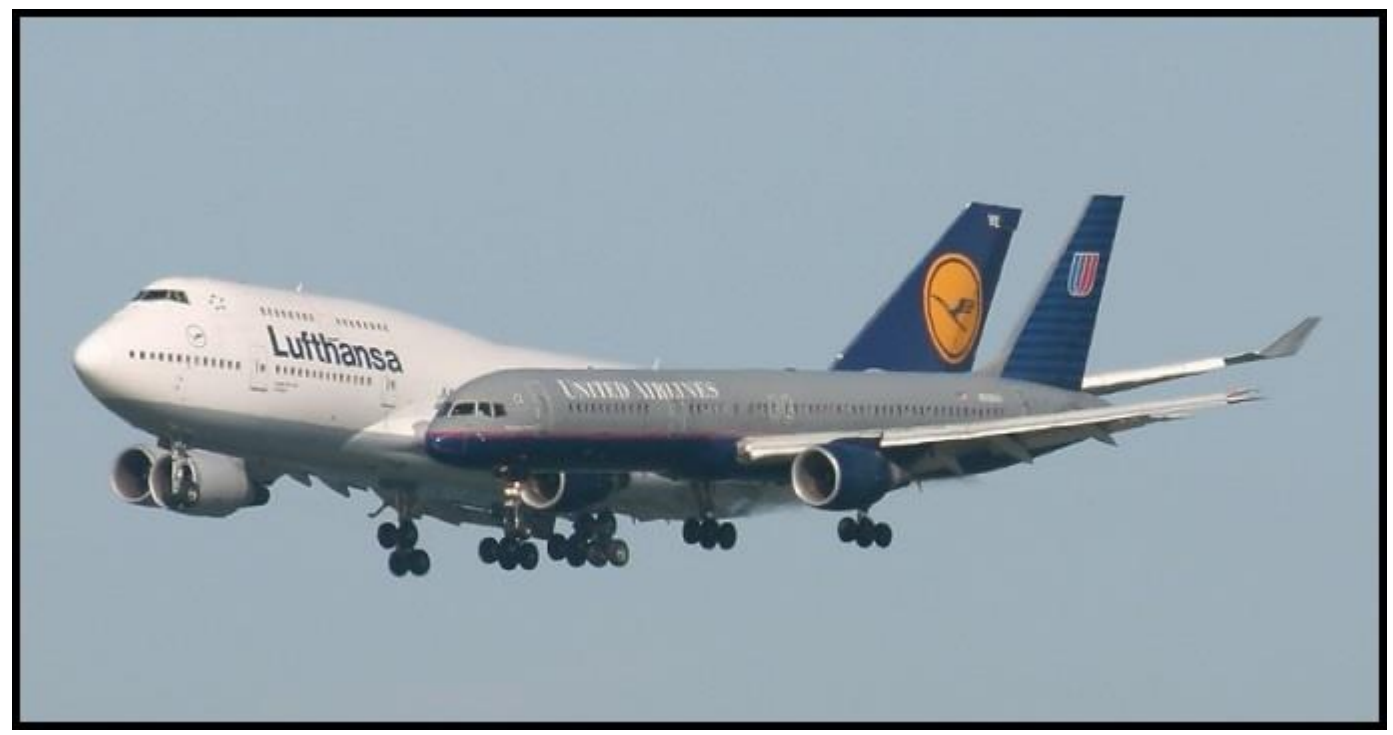

Figure 8. Optical illusion of Lufthansa 747-400 and United Airlines 757-200 on simultaneous approaches to runways $28 \mathrm{~L}$ and $28 \mathrm{R}$ at San Francisco (SFO). The distance offset is difficult to perceive, as there is a natural tendency to relate objects in terms of comparable relative size. In this case, however, the 747-400 is substantially larger than the 757-200, which is not apparent by visual comparison alone. This figure illustrates a similar optical illusion to that encountered by experimental participants in which size differential adversely affected observers' ability to accurately judge distances. Used with permission, courtesy of photographer Ben Wang, Airliners.net.

Proximity between aircraft and UAS platform created a strong perceptual illusion. At higher vertical angles of participant observation-particularly when the aircraft and sUAS intercepted overhead or were relatively close to the observer--participants tended to perceive a worse collision potential than what actually existed. This resulted in several participants indicating that they perceived the sUAS platform was actually co-altitude or above the height of the manned aircraft, despite having been briefed that the sUAS and aircraft were at fixed, deconflicted altitudes. Participant 8 explained, "When distances are large (greater than 500 feet), I had more confidence in estimates; when they are smaller (less than 200 feet) it was more difficult to estimate." This was a particularly interesting finding, considering that altitude differential was only peripherally studied in this research. The phenomenon was so powerful, it was experienced by almost every participant. Eight of the 10 participants reported experiencing this perception and it was encountered during 49\% $(n=19)$ of the 39 intercepts. Participant 5, an experienced commercially-certificated pilot, exclaimed, "this isn't 
right" during all four of the intercepts. He further stated that "the perception was overpowering," despite his unequivocal knowledge that the aircraft was flying 200 feet above the altitude of the sUAS. In several cases, this same perception was shared by the ground research observer, who noted the finding in his field notes.

Researchers took several photographs to depict the illusion. The best illustrative examples of this illusion are presented in Figures 9 and 10.

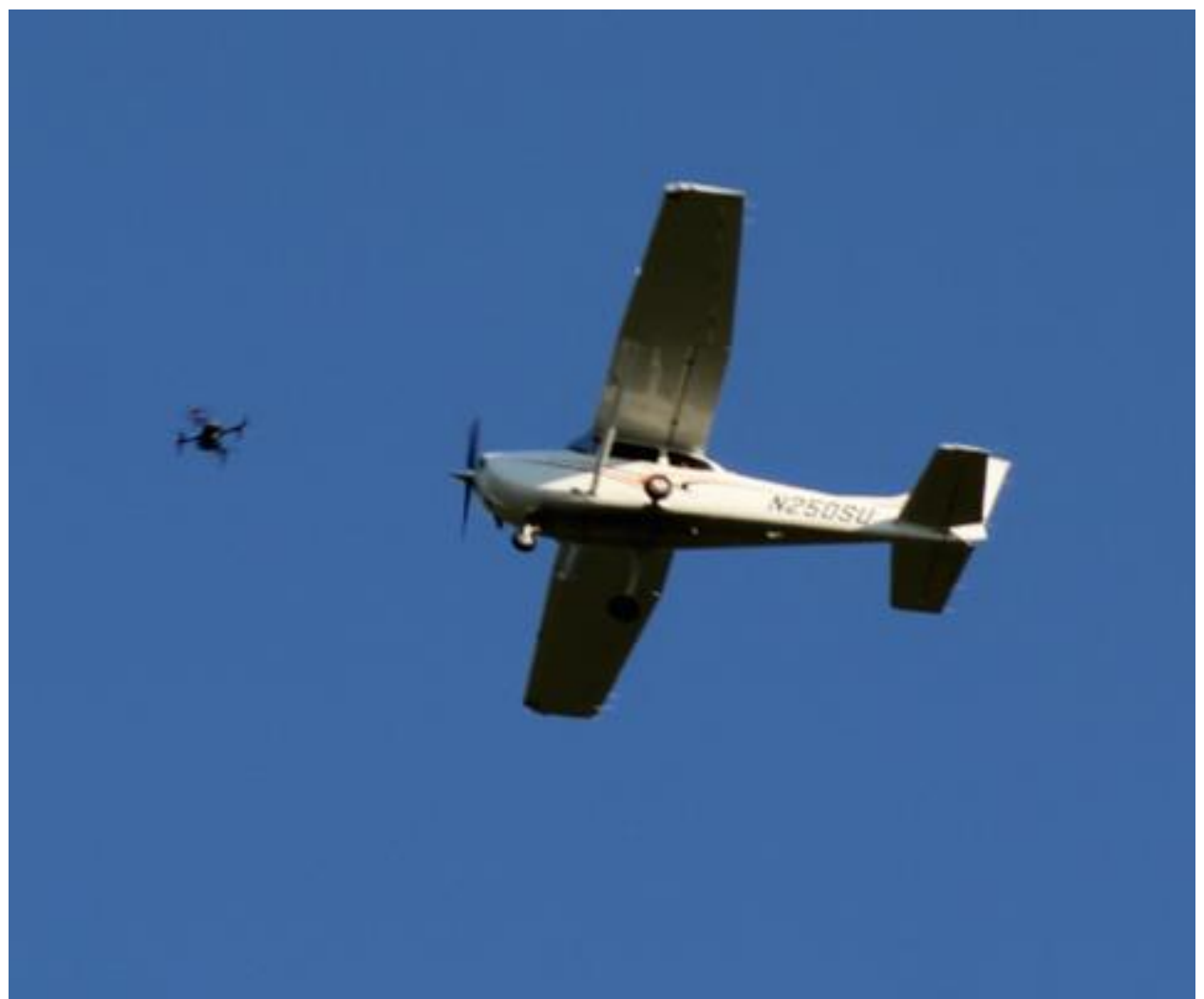

Figure 9. Ground observer image of Participant 2, Intercept 3. Participant 2 indicated that the sUAS appeared to be at a greater altitude than the aircraft. Small UAS and aircraft altitudes were fixed at 400 feet AGL and 600 feet AGL, respectively. 


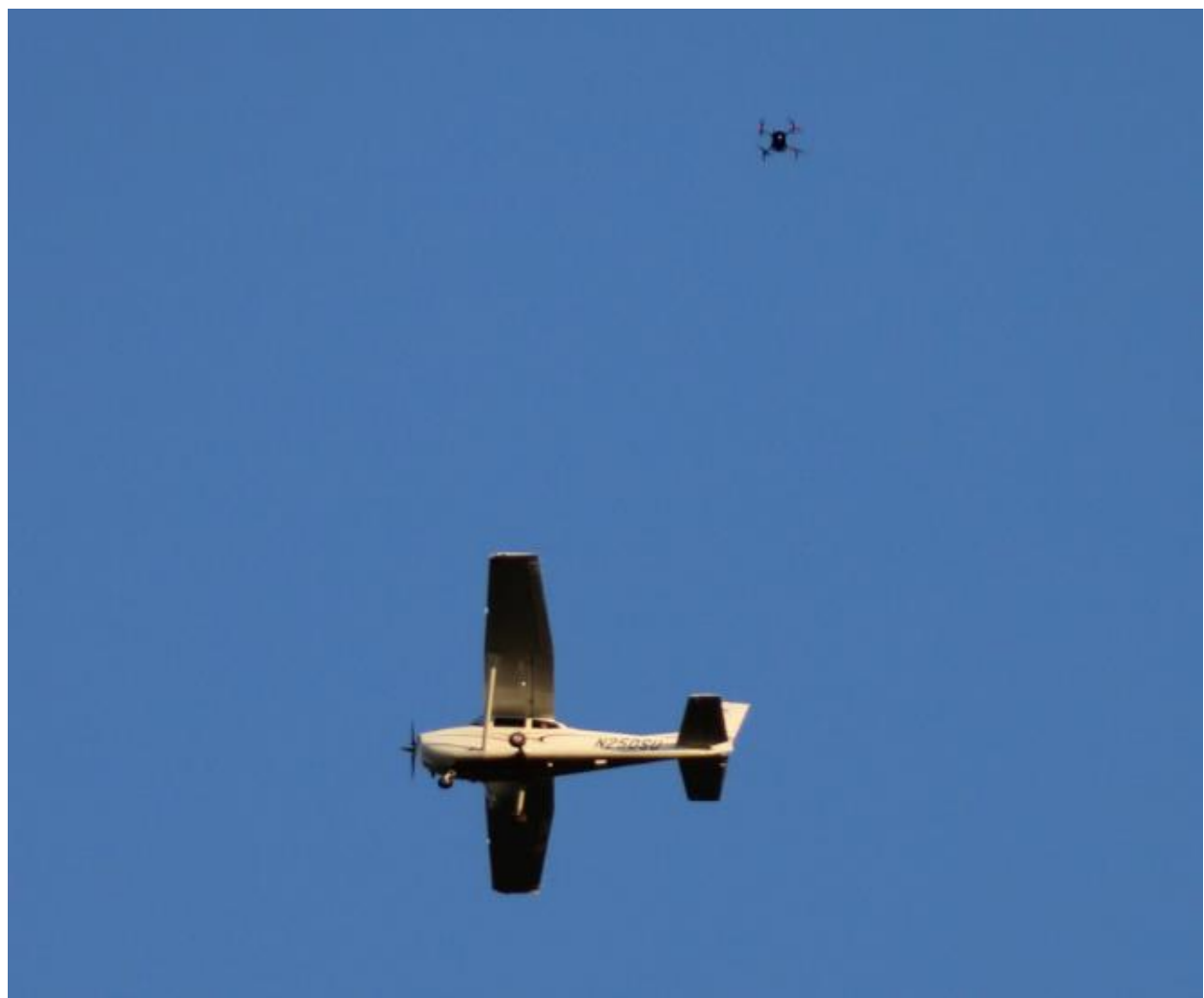

Figure 10. Ground observer image of Participant 3, Intercept 3. Participant 3 indicated that the sUAS appeared to be higher in altitude than the aircraft.

\section{Conclusions}

Research Question 1: At what range do visual observers reliably detect invasive aircraft?

Data suggests that visual observers reliably detected invasive aircraft auditorally at a mean range of 8,605.4 feet, and visually at a mean range of 8,618.6 feet. This equates to approximately 1.6 statute miles for both sensory modalities. While this conclusion provides some perspective to field performance of visual observer detection range, it should be taken with skepticism. Other factors are highly likely to affect visual observer performance. Aircraft size or surface area, aspect angle, aircraft reflectivity, light level, relative sun position or glare, sky contrast, visual obstructions, external aircraft lighting, and a myriad of other factors are likely to affect visual observer visual detection range. Similarly, observer audio detection range is likely to be affected by comparable conditions such as aircraft engine type, power setting, altitude, wind direction and speed, ambient noise and other related conditions. 
Small UAS operators can reasonably anticipate that visual observers can reliably detect aircraft at a maximum distance of 1.6 SM, under ideal conditions, but actual detection performance could be significantly reduced under less than ideal visual or auditory conditions.

Research Question 2: How accurate are visual observers at estimating distance to aircraft?

Crognale (2009) conclusively stated that visual observers were relatively poor at accurately estimating distance and altitude. This research further validates Crognale's conclusion. Visual observers were 2.5 times more likely to overestimate rather than underestimate distance. Even at close object distances, the variability between actual and estimated distance was relatively high at 290 feet. This conclusion is further supported by the qualitative responses from the participants.

Small UAS operators should not rely on visual observers alone to provide distance separation judgements, as the data suggests that visual observer capacity to accurately estimate distance is exceedingly poor. While this study did not directly assess altitude estimates, qualitative results further suggest that observer capacity to accurately assess altitude is similarly poor.

Research Question 3: Does the error between visual observer estimates and actual aircraft closure rate provide adequate time for a remote pilot to initiate evasive maneuvers before a potential collision?

This question ultimately forms the crux of this study, as the sole purpose of observers is to aid the remote pilot in detecting and sizing up potential collision threats. The data suggests that visual observer closure rate estimates are generally poor, and as previously indicated, demonstrated a tendency towards overestimation rather than underestimation. The mean estimation error was 17 seconds, which quantitatively does not sound substantial. When compared against the available mean response time of 54 seconds from initial aircraft detection to intercept, however, one can quickly see that there may not be a large margin for error to facilitate an evasive response. Moreover, a $\mathrm{C}-172 / \mathrm{S}$ is usually regarded as a relatively slow general aviation aircraft. Should the invasive aircraft be a faster single engine, multi-engine, or jet aircraft, available response time would be singificantly reduced. 
To illustrate the importance of this finding, the authors used the FAA's [Manned] Aircraft Identification and Reaction Time Chart as a basis for comparison (FAA, 2016).

Table 2

Manned Aircraft Identification and Reaction Time Chart

\begin{tabular}{ll}
\hline Event & Seconds \\
\hline See Object & 0.1 \\
Recognize Aircraft & 1.0 \\
Become Aware of Collision Course & 5.0 \\
Decision to Turn Left or Right & 4.0 \\
Muscular Reaction & 0.4 \\
Aircraft Lag Time & 2.0 \\
TOTAL & 12.5 \\
\hline
\end{tabular}

(FAA, 2016, p. 2)

According to the FAA, a pilot requires 12.5 seconds to detect and recognize an invasive flying object, assess its collision potential, make an avoidance decision, and initiate and complete an avoidance maneuver. While the process is not identical to that of a manned aircraft, remote pilots must follow a similar series of responses, but are further hampered with a communication requirement with their visual observers. Researchers suggest that this response process might appear similar to that presented in Table 3.

Table 3

Remote Pilot Invasive Aircraft Identification and Reaction Chart

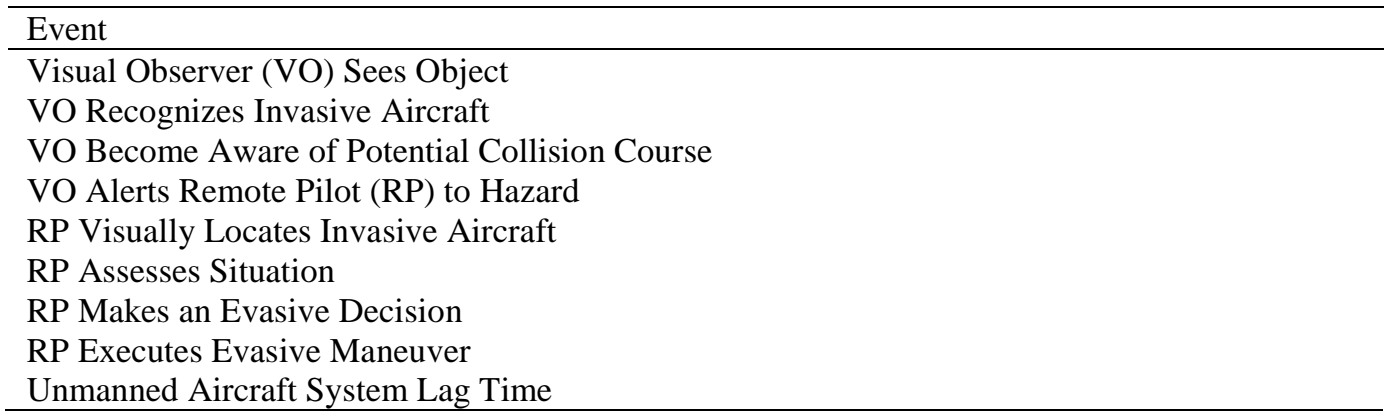

While it is currently unknown how long would be required to complete this proposed process, it is highly likely to exceed the duration of the FAA's midair collision timeline presented in Table 2. Conclusively, there is likely to be adequate time for a remote pilot to initiate evasive maneuvers before a potential collision with an invasive aircraft, provided the visual observer does not substantially delay communicating perceived collision potential to the remote pilot in a clear and 
succinct manner. The authors reocmmend additional research to better understand and evaluate the process and timeline associated with remote pilot evasive response.

Research Question 4: What human factors considerations affect visual observer detection and assessment of aircraft collision potential?

Qualitative responses made by the participants suggest that several visual or perceptive illusions may adversely affect visual observer performance. Size differential between the aircraft and sUAS platform was cited as one such impediment. While it may be difficult or impossible to remove the perceptual illusion created by this condition, visual observers may be able to better overcome the illusion by becoming familiar with the relative size and proportions of common aircraft and sUAS platforms. Visual observers may also find it beneficial to attempt visual size comparison against known components of either the aircraft or UAS platforms. For example, the wingspan of a Cessna 172 used during the experiment is $36 \mathrm{ft}$, whereas the diagnonal span of the DJI Matrice 100 (without props) is approximately $2.3 \mathrm{ft}$. See the examples in conjunction with Figure 11 for both illustration and practical explanation about how to apply the presented proportionality principles.

Distance estimation strategy. By multiplying the known width of the aircraft wingspan [36 ft] by the visible, observed lateral separation between the sUAS and the aircraft, the visual observer can better estimate the actual distance. In the example provided in Figure 11, the result is approximately 1.5 wingspans, or about $50 \mathrm{ft}$.

Altitude estimation strategy. The proportion of the visible sUAS width to that of known components of the small manned aircraft, such as the wingspan, can be used to assess the relative altitude of the sUAS to the manned aircraft. In the case of Figure 11, the sUAS width is visibly equal in length to approximately $1 / 6$ that of the manned aircraft's wingspan. By multiplying the known width of the aircraft wingspan [36 feet] by the visible proportion of sUAS [approximately 1/6], the visual observer can better assess perceptual distance errors. In this case, the result is $6 \mathrm{ft}$. Since $6 \mathrm{ft}$ is substantially larger than the known width of the Matrice 100 [actual width: 2.3 feet], then the visual observer can accurately determine that the sUAS must be closer in altitude than the aircraft.

Determining collision potential was reportedly a significant challenge for most participants. As previously stated, the ability of visual observers to accurately 
judge distance, altitude, and collision potential is extremely limited. It is recommended that remote pilots apply generous safety buffers to any information provided solely on the basis of visual observer reports. Remote pilots and visual observers should also become familiar with available aeronautical charts, UAS facility maps, and other sources of information that may alert airspace areas, obstacles, or manned air traffic paths that enhance the predictability of hazards and overall situational awarness.

Both remote pilots and visual observers should realize that the accuracy of relative position and collision potential judgements become generally worse at closer proximities. This effect is especially pronounced as aircraft and sUAS platforms intercept overhead or at only slight lateral offsets from visual observers.

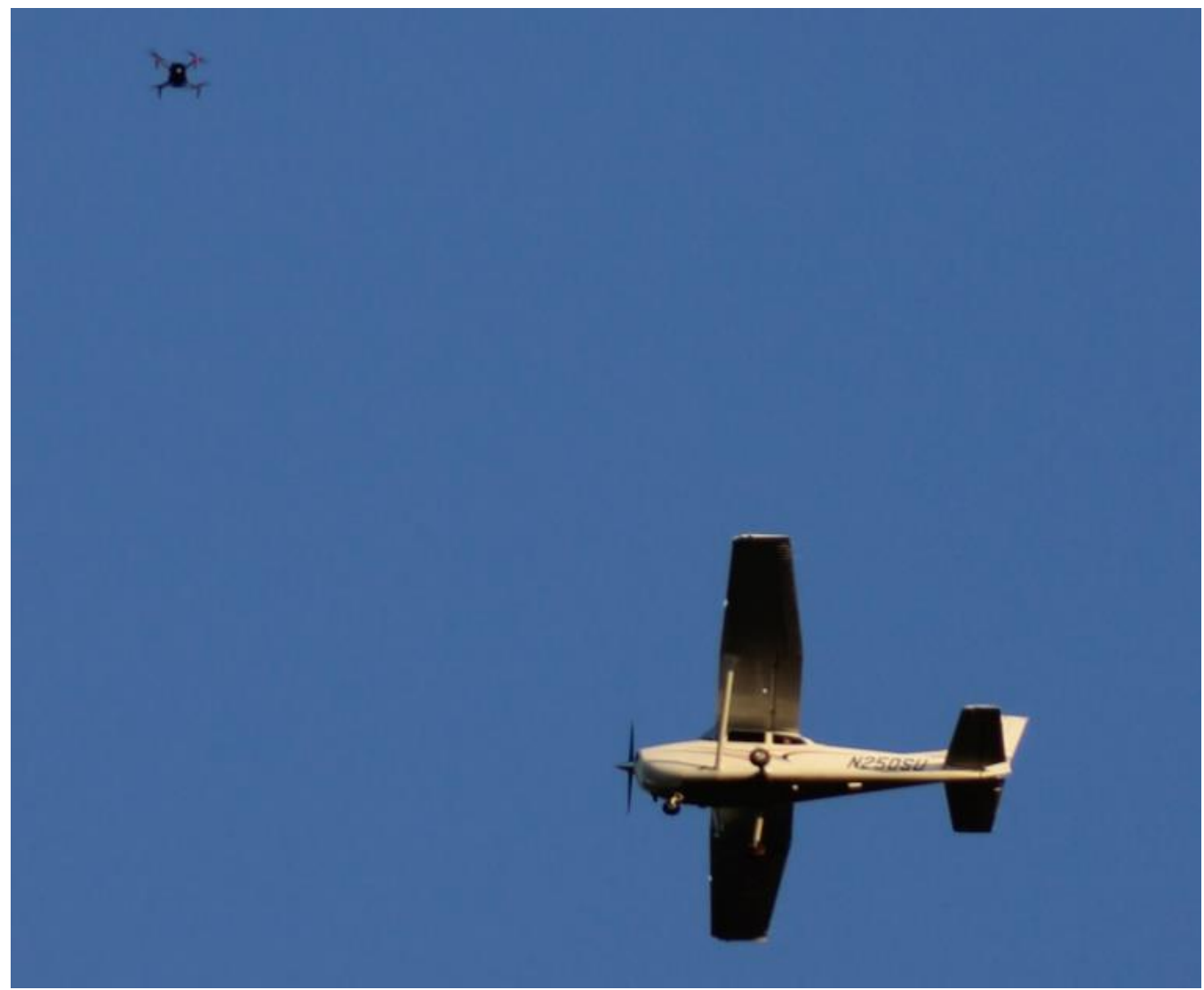

Figure 11. Ground observer image of Participant 1, Intercept 3. Visual observers can use relative width and proportion to better estimate distance and altitude information at close range. 


\section{Recommendations}

The authors recommend additional research be performed to better identify and codify the tasks associated with sUAS collision avoidance. Moreover, the authors suggest evaluating realistic invasive aircraft scenarios to better develop benchmarks for a sUAS evasive response timeline.

\section{Conclusion}

The use of visual observers is one of many tools available to remote pilots to comply with aviation regulations and ensure safety of flight. This study highlights the important human performance limitations associated with visual observer performance. Remote pilots should take heed to recognize and acknowledge these human factors limitations, and be wary of relying solely on this fallible modality of hazard detection. 


\section{References}

Crognale, M.A. (2009). UAS/UAV ground observer performance: Field measurements [FAA Technical Report: DOT/FAA/AR-10/1]. Washington, DC: Federal Aviation Administration Air Traffic Organization Planning, Office of Research and Technology Department.

Dolgov, I. (2016). Moving towards unmanned aircraft system integration into the National Airspace System: Evaluating visual observers' imminent collision anticipation during day, dusk, and night sUAS operations. International Journal of Aviation Sciences, 1(1), 41-56. https://www.ijas.us/index.php/issues/current-issues/issue-i-volume1/articles/moving-towards-unmanned-aircraft-systems-integration-intothe-national-airspace-system-evaluating-visual-observers-imminentcollision-anticipation-during-day-dusk-and-night-suas-operations-igordolgov

Federal Aviation Administration. (2016). Pilots' role in collision avoidance [Advisory Circular, AC 90-48D] Retrieved from http://www.faa.gov/ documentLibrary/media/Advisory_Circular/AC_90-48D.pdf

Small Unmanned Aircraft Systems, 14 CFR § 107 (2017).

Williams, K.W., \& Gildea, K.M. (2014). A review of research related to unmanned aircraft system visual observers [FAA/CAMI Report: DOT/FAA/AM-14/9]. Retrieved from http://www.faa.gov/data_research/ research/med_humanfacs/oamtechreports/2010s/media/201409.pdf 